\title{
A genomic region involved in the formation of adhesin fibers in Bacillus cereus biofilms
}

\author{
Joaquín Caro-Astorga, Alejandro Pérez-García, Antonio de Vicente and Diego Romero*
}

Departamento de Microbiología, Facultad de Ciencias, Instituto de Hortofruticultura Subtropical y Mediterránea "La Mayora" (IHSM-UMA-CSIC), Universidad de Málaga, Málaga, Spain

\section{Edited by:}

Javier Carballo, University of Vigo,

Spain

Reviewed by:

Akos T. Kovacs, Friedrich Schiller

University of Jena, Germany

Weifeng She, Johns Hopkins

University, USA

*Correspondence:

Diego Romero, Departamento de Microbiología, Facultad de Ciencias, Instituto de Hortofruticultura Subtropical y Mediterránea "La Mayora" (IHSM-UMA-CSIC), Universidad de Málaga, Bulevar Louis Pasteur-Campus Universitario de Teatinos s/n 29071 Málaga, Spain e-mail:diego_romero@uma.es
Bacillus cereus is a bacterial pathogen that is responsible for many recurrent disease outbreaks due to food contamination. Spores and biofilms are considered the most important reservoirs of $B$. cereus in contaminated fresh vegetables and fruits. Biofilms are bacterial communities that are difficult to eradicate from biotic and abiotic surfaces because of their stable and extremely strong extracellular matrix. These extracellular matrixes contain exopolysaccharides, proteins, extracellular DNA, and other minor components. Although B. cereus can form biofilms, the bacterial features governing assembly of the protective extracellular matrix are not known. Using the well-studied bacterium $B$. subtilis as a model, we identified two genomic loci in $B$. cereus, which encodes two orthologs of the amyloid-like protein TasA of $B$. subtilis and a SipW signal peptidase. Deletion of this genomic region in $B$. cereus inhibited biofilm assembly; notably, mutation of the putative signal peptidase SipW caused the same phenotype. However, mutations in tasA or calY did not completely prevent biofilm formation; strains that were mutated for either of these genes formed phenotypically different surface attached biofilms. Electron microscopy studies revealed that TasA polymerizes to form long and abundant fibers on cell surfaces, whereas CalY does not aggregate similarly. Heterologous expression of this amyloid-like cassette in a $B$. subtilis strain lacking the factors required for the assembly of Tas $A$ amyloid-like fibers revealed (i) the involvement of this $B$. cereus genomic region in formation of the air-liquid interphase pellicles and (ii) the intrinsic ability of TasA to form fibers similar to the amyloid-like fibers produced by its $B$. subtilis ortholog.

Keywords: Bacillus cereus, biofilm formation, amyloid, extracellular matrix, food-borne pathogens

\section{INTRODUCTION}

A major food-safety problem in developing countries is the contamination of fresh, stored and packaged food by bacteria that decrease the shelf life of the product and cause human poisoning (Burnett and Beuchat, 2001). Consumption of raw vegetables and fruits, milk, eggs, mildly cooked rice or pasta is typically associated with the most common outbreaks of poisoning (Carlin et al., 2000; Kamga Wambo et al., 2011). The symptoms of food poisoning can be mild, such as vomiting and diarrhea, or more severe, such as bacteremia; in severe cases, it can cause death of the patients. Bacterial strains of Escherichia coli, Salmonella, Enterococcus, Lysteria and Bacillus cereus are recurrent etiological agents of poisoning outbreaks (Berger et al., 2010). Epidemiological studies of these outbreaks have revealed that product contamination occurs before the manufacturing step. Irrigation with wastewater and the use of natural plant strengtheners lead to contamination of vegetables by enteropathogenic E. coli and Salmonella strains (Berger et al., 2010).

Several B. cereus strains are commonly observed as the etiological agents of poisoning outbreaks, severe bacteremia and septicemia (Bottone, 2010). B. cereus, a naturally inhabitant of soils, is frequently isolated from fresh vegetables and ready-to-eat vegetable-based food and is implicated in outbreaks of gastrointestinal diseases, abdominal pains, and watery diarrhea (Elhariry, 2011). B. cereus causes two main types of poisoning: emetic and diarrheic. Emetic poisoning is associated with production of cerulide, a lipophilic toxin. This toxin is extremely heat stable, and it can be produced in food contaminated by B. cereus cells. Notably, cerulide may persist in the body for a long period, affecting different organs and eventually leading to patient death (Thorsen et al., 2011). Diarrheic poisoning is caused by another group of toxic molecules: enterotoxin Hemolysin $\mathrm{BL}(\mathrm{HBL})$, the non-hemolytic enterotoxin (NHE) and cytotoxin (CytK). However, the specific role of each toxin in symptom development has not been elucidated. NHE and CytK are individually sufficient to induce diarrhea; however, it is not known whether HBL acts similarly. Similar to cerulide, HBL, NHE, and CytK can be produced in food contaminated with B. cereus cells; however, the sensitivity of these toxins to low $\mathrm{pH}$ and digestive proteases prevents the development of diarrheic symptoms. Therefore, poisoning occurs due to enterotoxin production in the small intestine by B. cereus cells or spores that have been ingested (McKillip, 2000).

Colonization and persistence of B. cereus cells in fresh vegetables and fruits are required for intoxication. B. cereus 
produces spores highly resistant to stressful environments and are able to survive heat, dry conditions, sanitation procedures, and food-processing treatments; and also aggregates in bacterial communities called biofilms (Ball et al., 2008; Shaheen et al., 2010; Elhariry, 2011). Studies on the related bacterial species B. subtilis revealed that biofilms are natural reservoirs of spores and are as recalcitrant as spores to eradication therapies (Branda et al., 2005). Biofilm formation requires (i) a complex regulatory pathway that coordinates gene expression with external environmental conditions and (ii) structural components involved in the assembly of a protective extracellular matrix (Romero, 2013; Vlamakis et al., 2013). In B. subtilis, the extracellular matrix is composed of exopolysaccharides, the hydrophobin protein BlsA and the amyloid-like protein TasA (Branda et al., 2004; Romero et al., 2010; Kobayashi and Iwano, 2012; Hobley et al., 2013; Romero, 2013). Studies on bacterial ecology have focused on amyloid proteins because: (i) they retain the morphological and biochemical features of their pathogenic siblings in humans (Fowler et al., 2007), (ii) they are involved in multiple functions relevant to bacterial physiology and ecology (Chapman et al., 2002; Epstein and Chapman, 2008; De Jong et al., 2009; Dueholm et al., 2010; Romero et al., 2010; Schwartz et al., 2012), and (iii) they undergo a complex program leading to fibrillation (Blanco et al., 2012). In B. subtilis biofilms, TasA amyloid-like fibers constitute the protein skeleton that directs the assembly of the extracellular matrix (Romero et al., 2010).

Although biofilm formation has been studied in detail in B. subtilis, not much is known about this developmental program in B. cereus. Separate studies revealed that specific $B$. cereus elements are involved in biofilm formation: notably, $\sin R$ and $\sin I$, spoOA or $a b r B$, major regulators controlling the developmental program ending in biofilm formation of $B$. subtilis has also been demonstrated to play similar roles in biofilm formation of $B$. cereus. The SinR regulon in a strain of $B$. thuringiensis closely related to $B$. cereus contains the loci $\operatorname{sip} W$-tas $A$, as it does in B. subtilis, but also the lipopeptide kurstakin, important for biofilm formation (Pflughoeft et al., 2011; Fagerlund et al., 2014). In addition, $B$. cereus appears to form wrinkly colonies and cell bundles in response to glycerol, manganese or milk, and this is proposed to be mediated by the kinase KinD as seen in B. subtilis (Shemesh and Chai, 2013; Pasvolsky et al., 2014). Other two major regulators of $B$. cereus with involvement in biofilm are $\mathrm{PlcR}$, the main virulence regulator, and CodY, a repressor of branched aminoacids, which points toward the inevitable connection of virulence with biofilm formation in this bacteria species (Hsueh et al., 2006; Lindback et al., 2012). Besides this knolowledge on the biofilm-dedicated regulatory pathways, other studies have revealed the relevance on motility on adhesion to abiotic surfaces, or the presence of extracellular DNA and other uncharacterized proteins or polysaccharides in the extracellular matrix of biofilms of B. cereus (Auger et al., 2009; Vilain et al., 2009; Houry et al., 2010; Karunakaran and Biggs, 2011).

Because amyloid-like fibers are important for biofilm formation in diverse bacterial species, we examined the role of a genomic region encoding two orthologs, TasA and CalY, of the B. subtilis TasA amyloid-like protein in biofilm formation by $B$. cereus. Using mutagenesis analysis, we revealed that this region is important for biofilm assembly in B. cereus CECT148. Electron microscopy analysis revealed the presence of TasA fibers on the B. cereus cell surfaces, similar to those formed by B. subtilis TasA. Furthermore, by heterologous expression of B. cereus alleles in B. subtilis mutants lacking different components required for amyloid-like fiber assembly, we observed that $B$. cereus TasA functions similar to the endogenous $B$. subtilis TasA protein: (i) it is involved in the formation of wrinkles in the air-liquid interphase pellicle, a visual feature of mature biofilms, (ii) the pellicles are positively stained with the amyloid-specific dye Congo Red and (iii) abundant and robust fibers are assembled on cell surfaces.

\section{MATERIALS AND METHODS BACTERIAL STRAINS AND CULTURE CONDITIONS}

The bacteria used in this study are listed in Table 1. Bacteria were routinely grown in Ty broth (1\% tryptone, OXOID), $0.5 \%$ yeast extract (OXOID), $0.5 \% \mathrm{NaCl}, 10 \mathrm{mM} \mathrm{MgSO}_{4}$, and $1 \mathrm{mM} \mathrm{MnSO}_{4}$. Biofilm assays were performed either in TY or MSgg broth (100 mM morpholinopropanesulfonic acid (MOPS) ( $\mathrm{pH}$ 7), $0.5 \%$ glycerol, $0.5 \%$ glutamate, $5 \mathrm{mM}$ potassium phosphate $(\mathrm{pH}$ 7), $50 \mu \mathrm{g} / \mathrm{ml}$ tryptophan, $50 \mu \mathrm{g} / \mathrm{ml}$ phenylalanine, $2 \mathrm{mM} \mathrm{MgCl}_{2}$, $700 \mu \mathrm{M} \mathrm{CaCl}_{2}, 50 \mu \mathrm{M} \mathrm{FeCl}_{3}, 50 \mu \mathrm{M} \mathrm{MnCl}_{2}, 2 \mu \mathrm{M}$ thiamine, and $1 \mu \mathrm{M} \mathrm{ZnCl}_{2}$ ) (Branda et al., 2001). Antibiotics were used when required at the following concentrations (final): MLS, $1 \mu \mathrm{g} / \mathrm{ml}$ erythromycin, $25 \mu \mathrm{g} / \mathrm{ml}$ lincomycin, spectinomycin $100 \mu \mathrm{g} / \mathrm{ml}$, chloramphenicol $5 \mu \mathrm{g} / \mathrm{ml}$, and kanamycin $10 \mu \mathrm{g} / \mathrm{ml}$.

\section{RNA PURIFICATION AND RT-PCR}

A $3 \mathrm{ml}$ culture of $B$. cerues CECT148 in LB was growth without agitation at $30^{\circ} \mathrm{C}$. After $24 \mathrm{~h}$, the tube was vortexed to resuspend the ring of biomass adhered to the wells of the tube and centrifuged at $7000 \mathrm{~g} 1 \mathrm{~min}$ to collect cells. The cells were washed and lysed in $1 \mathrm{ml}$ BirnBoim A solution (10\% sucrose; $10 \mathrm{mM}$ TrisHCl, pH8.1; $10 \mathrm{mM}$ EDTA; $50 \mathrm{mM} \mathrm{NaCl}$ ), supplemented with $20 \mu \mathrm{g} / \mathrm{ml}$ lysozyme from chicken egg white (Sigma) for $30 \mathrm{~min} 37^{\circ} \mathrm{C}$, and eventually sonicated (power discharge $0.5 \mathrm{~s}$, pause $0.5 \mathrm{~s}$, amplitude $20 \%$ and 20 pulses). Cells were pelleted at $7000 \mathrm{~g} 1 \mathrm{~min}$, and the pellet resuspended in $1 \mathrm{ml}$ of Trizol (Trireagent, Trisure) with $10 \mu \mathrm{l}$ of proteinase $\mathrm{K}$, and incubated at $60^{\circ} \mathrm{C} 20 \mathrm{~min}$. After that, $200 \mu \mathrm{l}$ of chloroform were added to the sample, mixed inverting the tube several times and centrifuged. Supernatant over the interface containing nucleic acids were collected carefully without disrupting the interphase. The subsequent steps for purification of RNA was performed using a commercial kit (Nucleospin RNA Plant, Macherey-Nagel). The integrity of the RNA extraction was tested by electrophoresis in agarose gel and cDNA was obtained using Titan One RT-PCR System (Roche). To prove which genes constitute an operon we performed PCR with cDNA as template using primers between genes to test which are transcribed in the same RNA molecule; and primers inside each gene to test if they were expressed. Genomic DNA was purified using the commercial kit UltraClean Microbial DNA Isolation of MOBIO Laboratories. Positive controls for each primer pair were included using genomic DNA as template and for negative controls RNA extraction as template to 
Table 1 | Strains used in this study.

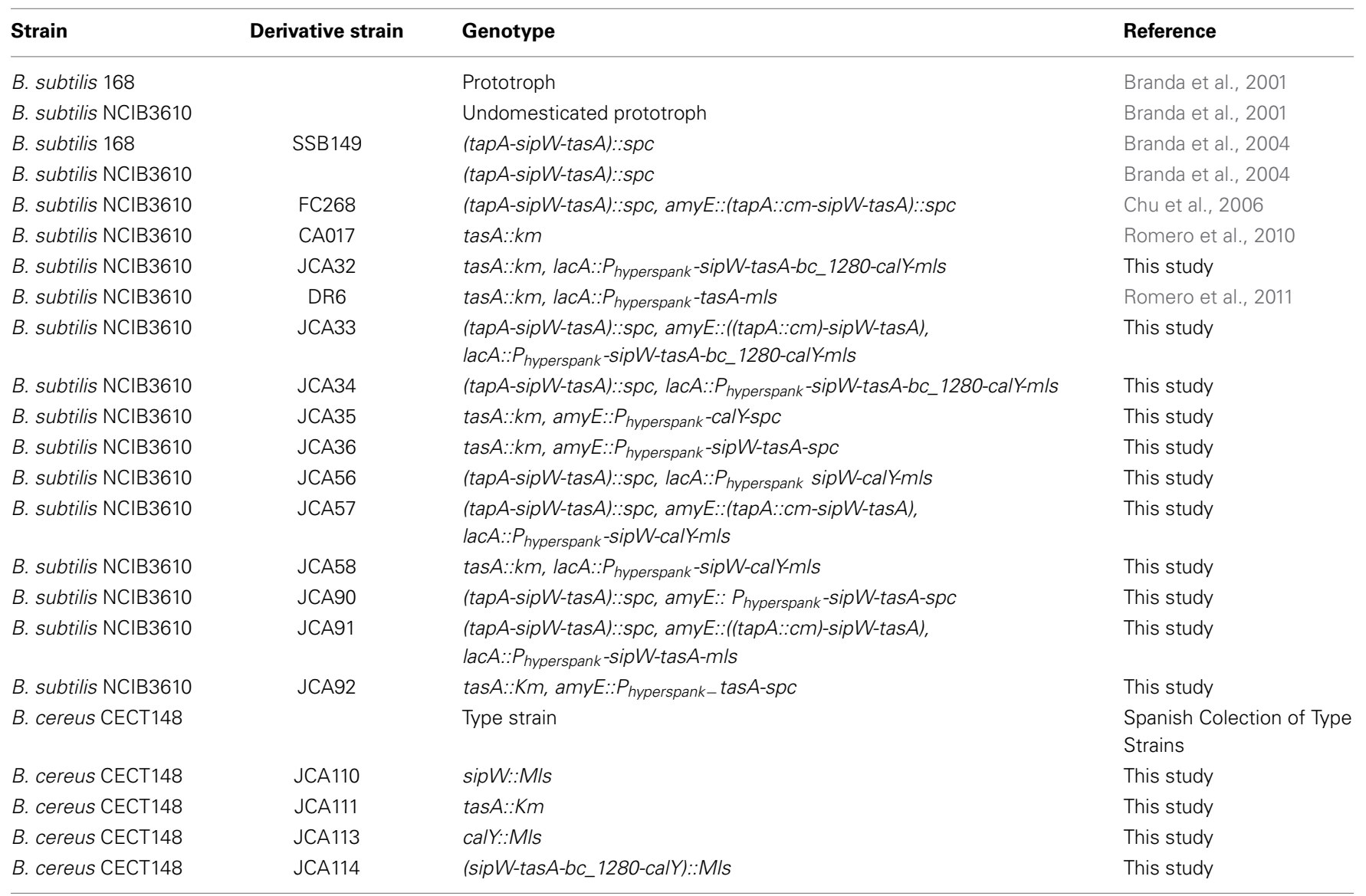

ensure that RNA extraction was not contaminated with genomic DNA. (Specific primers are specified in Table S1).

\section{CONSTRUCTION OF B. CEREUS MUTANTS}

$B$. cereus mutants were obtained by electroporation using derivatives of the plasmid pMAD (Arnaud et al., 2004). Primers used to generate the mutagenesis constructs are listed in Table S1. The constructs were created by joining PCR, as previously described (López et al., 2009). In the first step, regions flanking the target genes and antibiotic-resistance cassettes were amplified separately, purified, and used for the joining PCRs. These PCR products were digested with enzymes BamHI and NcoI and cloned into the pMAD vector digested with the same enzymes (Arnaud et al., 2004). The resulting suicide plasmids were used to transform B. cereus electrocompetent cells as described previously (Pflughoeft et al., 2011). Electroporations were performed with $4 \mu \mathrm{g}$ of plasmids and $100 \mu \mathrm{L}$ of electrocompetent B. cereus in 0.2-mm cuvettes using the following electroporation parameters: voltage $2500 \mathrm{kV}$, capacitance $25 \mu \mathrm{F}$, resistance $350 \Omega$. The electropored cells were seeded in LB plates supplemented with X-Gal and erythromycin for $72 \mathrm{~h}$ at $30^{\circ} \mathrm{C}$. Blue colonies were selected and restreaked as previously described to trigger allele replacement (Arnaud et al., 2004). Finally, white colonies that were sensitive to MLS were selected, and deletion of the target gene was verified by colony PCR analysis and sequencing of the amplicons.

\section{HETEROLOGOUS EXPRESSION OF B. CEREUS ALLELES IN B. SUBTILIS MUTANTS}

B. cereus alleles were amplified with specific primers (Table S1), digested and cloned into the integrative plasmid pDR111 (for ectopic integration at the amyE locus), digested with the same enzyme. When required, fragments containing the $P_{\text {hyperspank }}-$ promoter and the inserts were sub-cloned into the integrative plasmid pDR183 for ectopic integration at the lacA locus (López et al., 2009; Romero et al., 2011). The resulting integrative plasmids were used to transform B. subtilis 168 by natural competence; subsequently, using generalized transduction with Spp1 phages, the constructs were introduced into the recipient B. subtilis 3610 strains (Romero et al., 2011). The transformants were selected by antibiotic resistance and tested by PCR.

\section{BIOFILM ASSAYS}

B. subtilis biofilm formation was analyzed in MSgg medium (Branda et al., 2006). For pre-cultures, each strain was grown in $\mathrm{LB}$ agar with the required antibiotic at $37^{\circ} \mathrm{C}$ for $8 \mathrm{~h}$. A colony was resuspended in $1 \mathrm{ml}$ of MSgg, and $10 \mu \mathrm{l}$ was used to inoculate $1 \mathrm{ml}$ of MSgg in 24-well plates, and the plates were incubated without agitation at $30^{\circ} \mathrm{C}$. The pellicles were examined for the presence of wrinkles, a morphological feature of mature B. subtilis biofilms (Branda et al., 2006). 
B. cereus biofilm formation was monitored by testing bacterial adhesion to abiotic surfaces and staining with crystal violet (O'toole et al., 1999). Cultures were grown in TY at $30^{\circ} \mathrm{C}$ without agitation. One milliliter of a $1 \%$ solution of crystal violet in water was added to each well of a 24-well-plate. After 5 min of incubation, the plates were rinsed five times by immersion in tap water and were left inverted to dry on the bench for at least $45 \mathrm{~min}$. The crystal violet was then resuspended with $50 \%$ acetic acid. The resuspended solution was diluted $1 / 10$, and the absorbance was measured at $595 \mathrm{~nm}$.

\section{CONGO RED ASSAY}

Staining of B. subtilis pellicles with the amyloid dye Congo Red was performed as described above but using Ty medium supplemented with Congo Red and Coomassie Brilliant Blue G at final concentrations of 20 and $10 \mu \mathrm{g} / \mathrm{ml}$; the dyes were filtered and added to autoclaved Ty medium (Romero et al., 2010). The same procedures were used to stain $B$. cereus pellicles but grown in $4.5 \mathrm{~cm}$ diameter plates.

\section{IMMUNOLABELING AND TRANSMISSION ELECTRON MICROSCOPY ANALYSIS}

Each strain was grown in LB agar with the required antibiotic at $37^{\circ} \mathrm{C}$ for $8 \mathrm{~h}$. A colony was resuspended in $1 \mathrm{ml}$ of Ty (B. cereus strains) or MSgg (B. subtilis strains) and $10 \mu \mathrm{l}$ was inoculated in $1 \mathrm{ml}$ of Ty or MSgg in 24-well plates; the plates were incubated without agitation at $30^{\circ} \mathrm{C}$ for $24 \mathrm{~h}$. Cooper Grids for TEM were deposited on the air-liquid interface and incubated overnight. The grids were contrasted using 1\% uranyl acetate for $2 \mathrm{~min}$, rinsed by submersion in distilled water 2 min twice and then dried prior to examination. For immunolabeling assay, samples were floated on blocking buffer ( $1 \%$ non-fat dry milk in PBS with $0.1 \%$ Tween 20 ) for $30 \mathrm{~min}$, on anti-TasA of B. subtilis 1:150 for $2 \mathrm{~h}$, rinsed in PBST $30 \mathrm{~min}$ with a buffer change every $5 \mathrm{~min}$, floated in goat-antirabbit $40 \mathrm{~nm}$ gold secondary antibody (TedPella) $1: 50$ at $37^{\circ} \mathrm{C} 1 \mathrm{~h}$, rinsed in PBST and in ultrapure water four times for $5 \mathrm{~min}$ each. Samples were dried at RT and contrasted as previously described. Samples were visualized and photographed in a JEOL JEM-1400 transmission electron microscope.

\section{RESULTS}

\section{B. CEREUS ENCODES TWO ORTHOLOGS OF THE B. SUBTILIS AMYLOID-LIKE PROTEIN TASA}

B. subtilis biofilms are mainly composed of exopolysaccharides and the protein TasA. TasA can polymerize to form fibers that are morphologically and biochemically similar to amyloid proteins (Romero et al., 2010). A specific chromosomal region in B. cereus is similar to that of $B$. subtilis implicated in biofilm formation; this region encodes an ortholog of sipW and two orthologs of tas $A$ (tas $A$ and $c a l Y$ ). Using the genome sequence of the type strain $B$. cereus ATCC 14579 as reference, we identified all the genes of this region on B. cereus CECT148 (Figure 1A). It is important to mention, that an additional putative ortholog $\left(b c \_4868\right)$ with $29 \%$ identity to TasA of B. subtilis can be identified in the genome of $B$. cereus ATCC14579. This gene is predicted to encode a putative protease, and localized in the genome close to other protease-encoding gene, but we did not include it in our analysis.

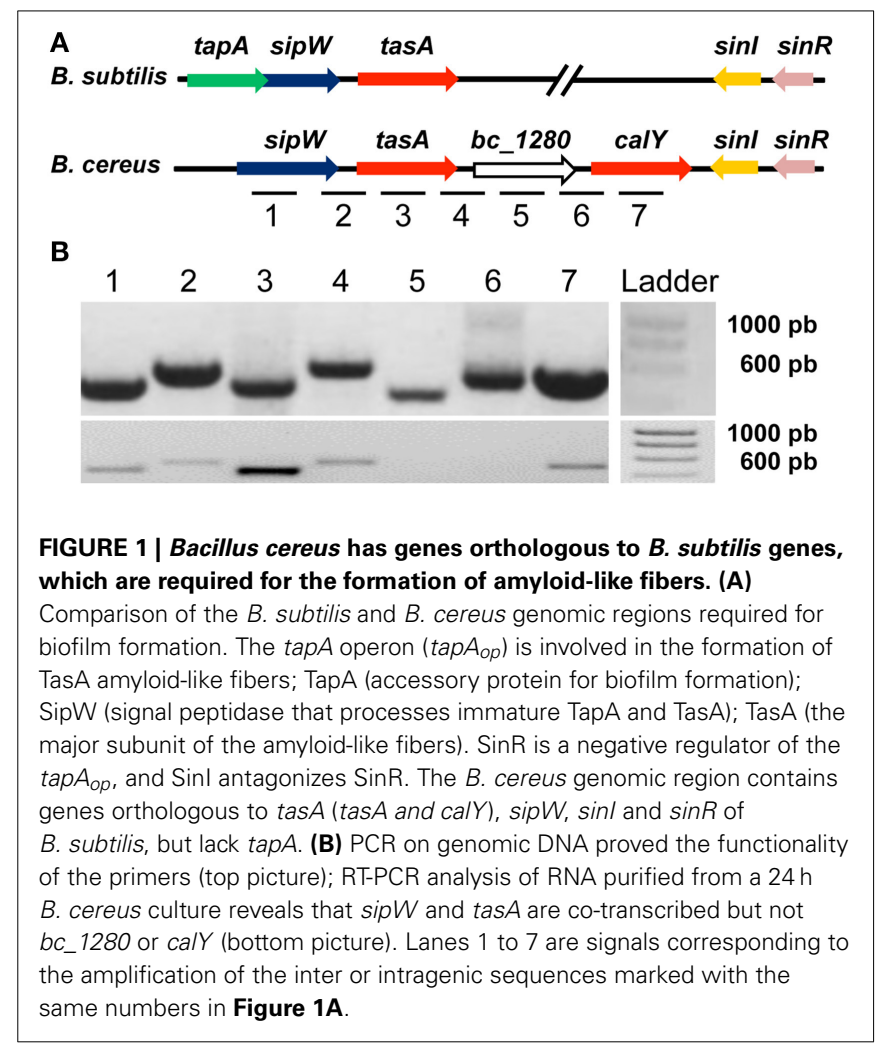

In $B$. subtilis, the genes tap $A$, sipW and tas $A$ constitute an operon $\left(\operatorname{tap} A_{o p}\right)$ and we proposed that this organization is conserved in $B$. cereus. To test this hypothesis, we extracted RNA from a 24-h culture of $B$. cereus and performed RT-PCR analysis using primers specific to each gene; we examined the expression of these genes to test whether they were transcribed together. First we confirmed the primers worked properly doing PCR on genomic DNA of B. cereus CECT148 (Figure 1B top picture). In the RT-PCR analysis we observed that sip $W$ and tas $A$ constitute an operon; the locus $b c \_1280$ was not expressed under our experimental conditions, and calY was expressed independently (Figure 1B bottom picture).

\section{SipW-tasA AND CaIY ARE INVOLVED IN B. CEREUS BIOFILM FORMATION}

Because tap $A_{o p}$ is important for the assembly of biofilms in $B$. subtilis, we examined the role of sip $W$-tas $A$ and calY in $B$. cereus biofilm formation. Biofilms were visualized by performing crystal violet staining on the biomass adhered to well surfaces (Figure 2). After $24 \mathrm{~h}$ of growth, wild-type cells formed visible rings, which grew in thickness up to $72 \mathrm{~h}$ (Figure $2 \mathrm{~A}$ top pictures). At all these stages, the rings of adhered biomass were strongly stained with crystal violet (Figure 2A bottom pictures, and Figure 2B). Deletion of the sipW-to-calY region prevented the formation of similar biomass rings; a mutation in sipW caused the same phenotype. SipW is a signal peptidase involved in TasA processing, which facilitates efficient secretion of TasA from B. subtilis cells (Stover and Driks, 1999b); mutation of sipW eliminated biofilm formation ability. Notably, deletion of tas $A$ conferred an unexpected phenotype: compared to the wild type strain, a 


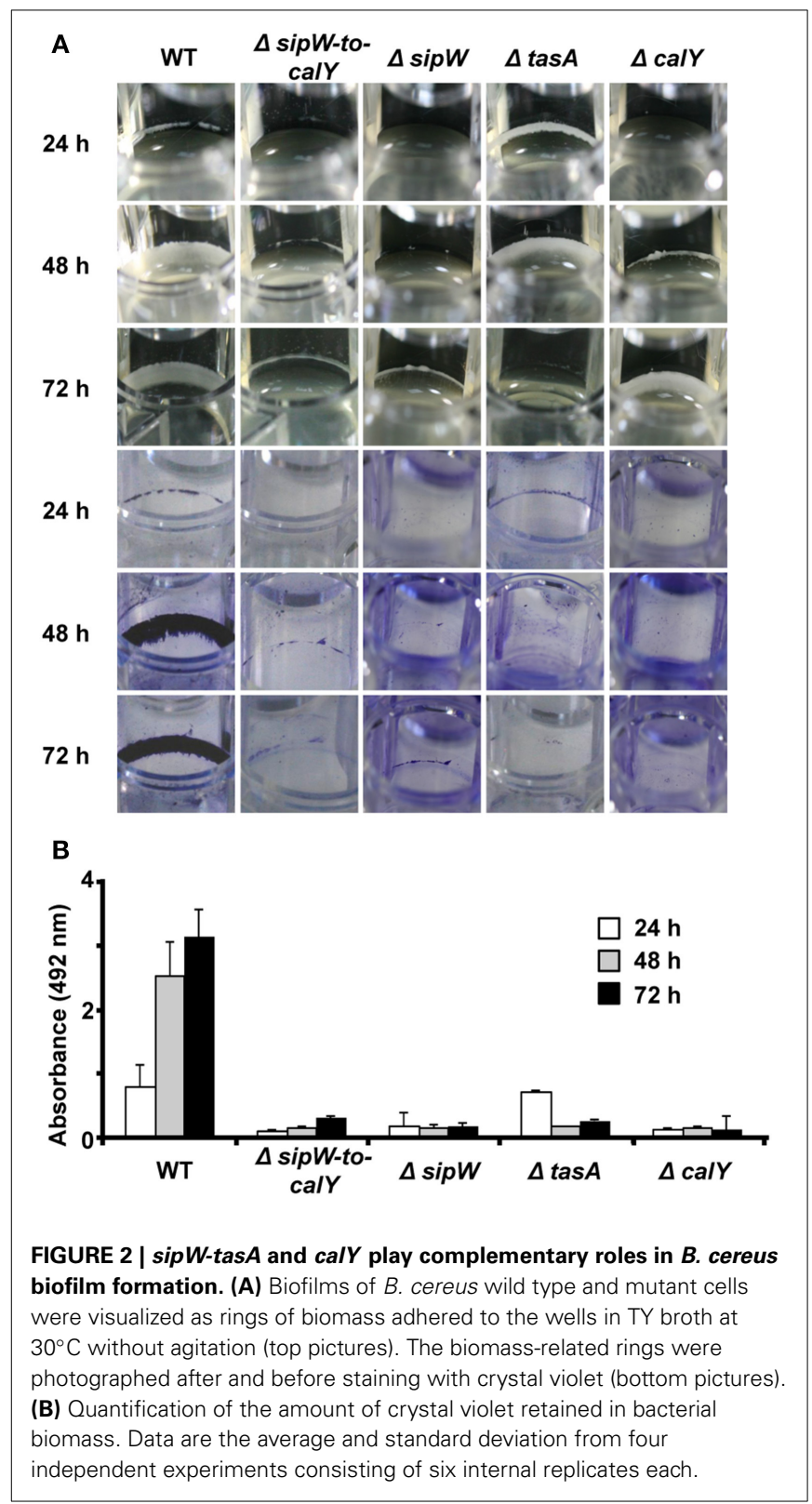

thicker biomass ring was observed in the $\operatorname{tas} A$ deletion strain at $24 \mathrm{~h}$, which disappeared at $72 \mathrm{~h}$. Upon staining with crystal violet, the biomass was extruded from the wells at each stage. Deletion of calY caused a different phenotype: the biomass adhered to the wells was thicker after $72 \mathrm{~h}$ of growth; however, similar to the phenotype of the tas $A$ mutant, the biomass did not bind tightly to the wells upon crystal violet staining. These observations revealed that this region is important for biofilm formation in B. cereus and that tas $A$ and calY potentially participate at different stages of biofilm formation, including initial attachment and maturation.

\section{TasA FORMS MORE ABUNDANT FIBERS THAN CaIY IN B. CEREUS BIOFILMS}

The formation of amyloid-like fibers in B. subtilis requires the function of two proteins, TasA and the accessory protein TapA
(Romero et al., 2014). Bioinformatics analysis revealed that at the amino acid level, TasA and CalY share only 31 and 32\% identity to $B$. subtilis tasA, respectively, but share $61 \%$ identity with each other. Based on these observations and the absence of a TapA ortholog in B. cereus, we proposed that TasA and CalY do not form fibers. To test this hypothesis, we use transmission electron microscopy to analyze $B$. cereus biomass adhered to wells (Figure 3, top row). Contrary to our hypothesis, B. cereus cells appeared highly decorated with fibers; however, cells with a deletion of this genomic region $(\Delta$ sipw-to-calY) or a mutation in sipW $(\Delta \operatorname{sip} W)$ did not form fibers. Consistent with our previous biofilm experiments (Figure 2), cells of the single $\triangle$ tas $A$ mutant, which expressed calY, produced thin and less abundant fibrils compared to the wild type. However, $\Delta$ calY mutant cells, which expressed $\operatorname{tas} A$, formed abundant fibers on their surfaces, similar to the wild type. To confirm that TasA or CalY formed fibers, we performed immunoelectron microscopy using antiTasA antibodies raised against B. subtilis TasA (Figure 3, bottom row). The cross immunereaction of $B$. cereus TasA with anti-TasA antibodies of $B$. subtilis was previously demonstrated (Pflughoeft et al., 2011). The fibers observed in wild type, $\Delta$ tas $A$ and $\Delta c a l Y$ $B$. cereus cells immunoreacted with the anti-TasA antibodies; however, no signal was observed in $\Delta$ sipW-to-calY or $\Delta$ sipW mutant cells.

Because B. cereus TasA forms fibers similar to B. subtilis TasA, we proposed that $B$. cereus biofilms would bind to amyloid dyes such as Congo Red. We performed biofilm experiments in 4.5$\mathrm{cm}$-diameter plates containing TY supplemented with Congo Red (Figure 4). Similar to the experiments performed with microtiter plates, no pellicles were observed during the initial stages of growth; however, after 5 days, a thin pellicle was visible in the $B$. cereus wild-type and $\Delta$ calY strains but not in $\Delta$ sipW-to-calY, $\triangle$ sipW or $\triangle$ tas $A$ mutants. When the medium was removed, the remaining pellicle appeared stained with Congo Red. These observations suggested that (i) TasA fibers are more important than CalY fibers for producing pellicles in the air-liquid interphase and (ii) B. cereus TasA fibers stain similarly to TasA amyloid-like fibers of B. subtilis.

\section{EXPRESSION OF THE B. CEREUS sipW-TO-caIY REGION RESCUES PELLICLE FORMATION IN B. SUBTILIS}

Our results suggested that the sip $W$-tas $A$ and calY are involved in $B$. cereus biofilm formation and that TasA is important for assembly of pellicles in the air-liquid interphase. Therefore, we proposed that $B$. cereus TasA might functionally replace $B$. subtilis TasA. B. subtilis forms wrinkly pellicles in the air-liquid interphase; this property facilitates identification of mutants that disrupt assembly of the normal architecture. In B. subtilis, deletion of TasA leads to a defect in assembling wrinkly pellicles (Branda et al., 2006). Therefore, we performed heterologous expression of the respective B. cereus loci (referred to as allele $e_{B c}$ ) in B. subtilis and examined the pellicle phenotype (Figure 5) and the adhesion to abiotic surfaces (Figure 6). The constructs were ectopically integrated at the amyE or lacA locus of B. subtilis, and their expression was driven by an IPTG-inducible promoter to bypass any potential regulatory processes associated with their native promoters. 

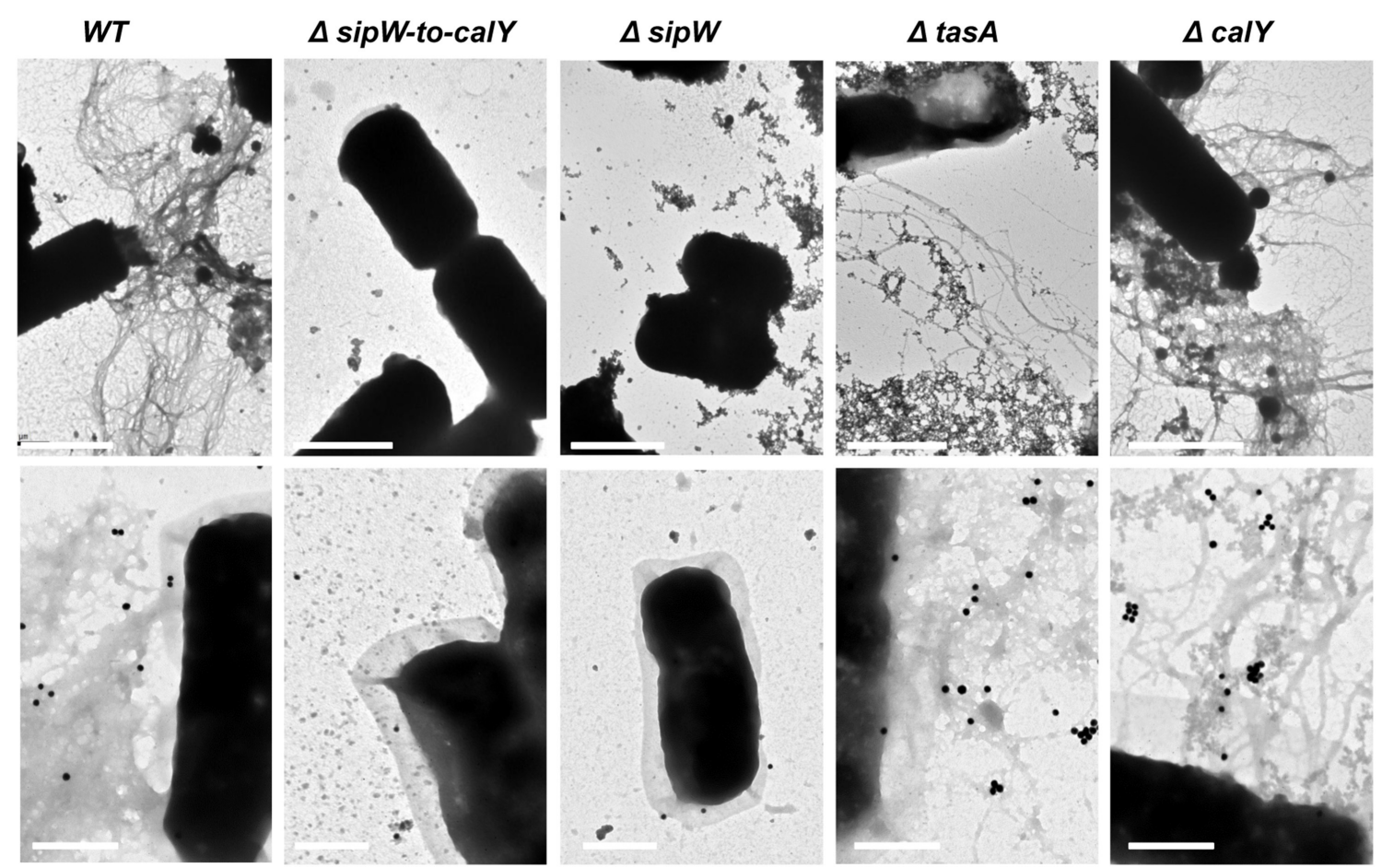

FIGURE 3 | TasA forms more abundant fibers than CalY in B. cereus. Biomass rings of $B$. cereus strains were isolated after $24 \mathrm{~h}$ of growth, contrasted with uranyl acetate and analyzed using transmission electron microscopy (top row), or immunolabeled with primary anti-TasA antibodies (1:150) and secondary antibody conjugated to $40 \mathrm{~nm}$ gold particles $(1: 50)$ before contrasting with uranyl acetate and visualization (bottom row). Bars equal $1 \mu \mathrm{m}$ (top row images) or $0.5 \mu \mathrm{m}$ (bottom row images).

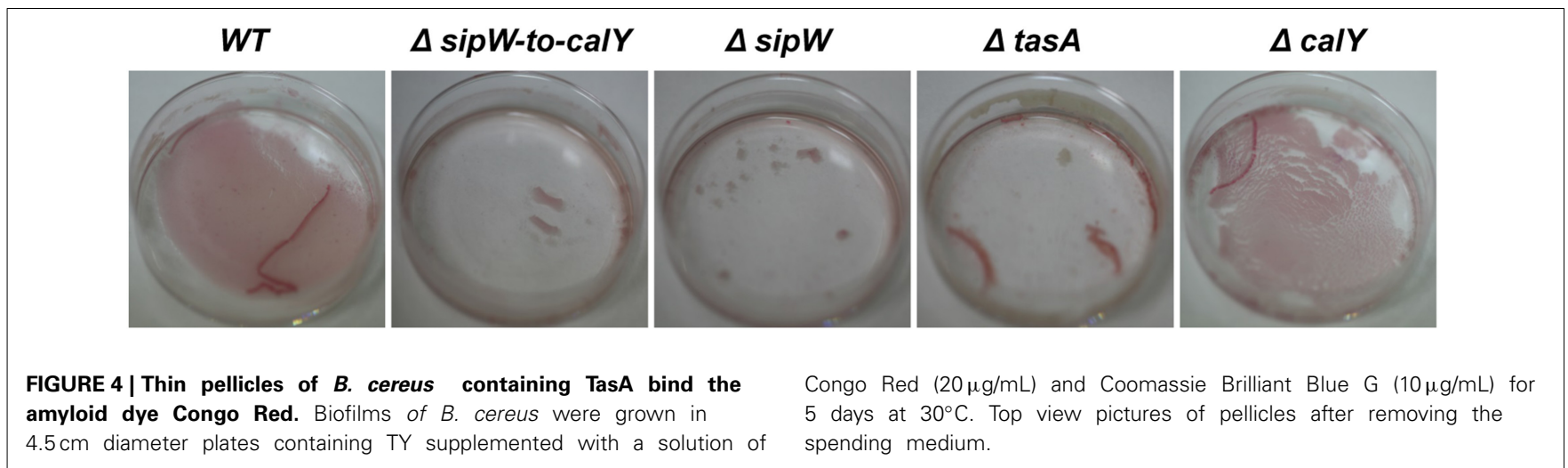

To examine the role of TasA in pellicle formation, we complemented a $B$. subtilis mutant lacking the tapA operon $\left(\triangle \operatorname{tap} A_{o p}\right)$ with the $B$. cereus sipW-to-calY chromosomal region (sipW-to$\left.c a l Y_{B c}\right)$. Our RT-PCR analysis indicated that calY is a monocistronic gene independent of sipW-tas A (Figure 1B), thus in this construct $\left(P_{\text {hyperspank }}\right.$-sipW-to-cal $\left.Y_{B c}\right)$ the expression of calY must rely under the control of its own promoter. As expected, this $B$. cereus construct rescued the formation of wrinkly pellicles, which resembled wild-type B. subtilis pellicles (Figure 5); furthermore, this construct notably enhanced the adhesion to the well surfaces in crystal violet assays (Figures 6A,B, top row). Therefore, the B. cereus sipW-to-calY region is involved in pellicle formation. Next, we examined the specific roles of tas $A$ and calY in pellicle formation. Expression of either tasA or calY failed to restore pellicle formation in the B. subtilis tasA mutant strain ( $\triangle$ tas $A$ ) (Figure S1, top and middle rows). We then tested whether these loci must be expressed with the cognate sip $W$ gene of B. cereus. Expression of sipW-tasA in the B. subtilis $\triangle$ tas $A$ mutant strain rescued pellicle formation (Figure 5, bottom row) and adhesion to abiotic surfaces, although less efficiently than that complementation with sipW-to-calY (Figures 6A,B bottom row). Notably, expression of the sipW-calY construct failed to restore pellicle formation (Figure 5, bottom row) or bind to abiotic surfaces (Figures $6 \mathbf{6}, \mathbf{B}$ ). Because the $B$. subtilis $\triangle$ tasA mutant 


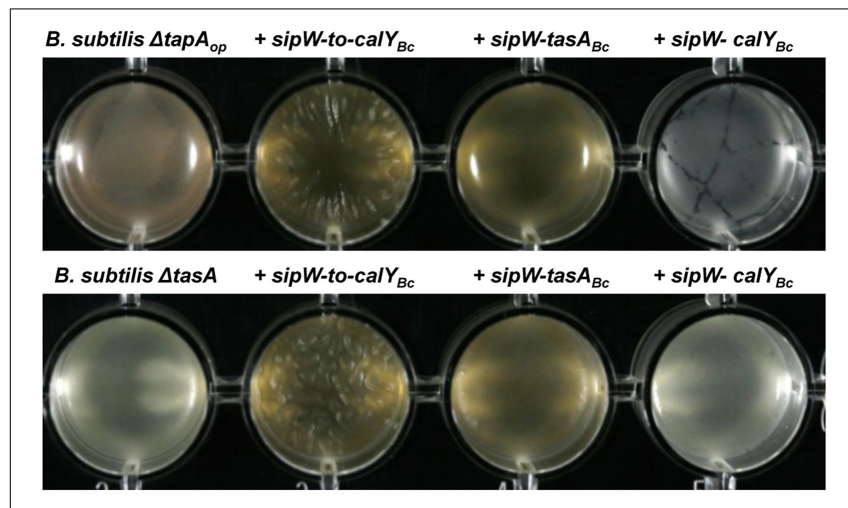

FIGURE 5 | Heterologous expression of B. cereus sipW-to-tas $A$ or $\operatorname{sip} \boldsymbol{W}$-tas $A$ in $B$. subtilis rescues pellicle formation. $B$. cereus alleles were ectopically integrated at the lacA or amyE locus of $B$. subtilis mutants lacking the entire $\operatorname{tap} A$ operon ( $B$. subtilis $\triangle \operatorname{tap} A_{o p}$ ) or lacking tas $A$ alone (B. subtilis $\triangle$ tas $A$ ); expression of these alleles was driven by an IPTG-inducible promoter. Biofilm experiments were performed in static cultures in MSgg broth and induced with 1 mM IPTG in 24-well plates. Top view of pellicles were photographed after $48 \mathrm{~h}$ of incubation in MSgg broth at $30^{\circ} \mathrm{C}$.

strain encodes the tapA gene, which is required to form pellicles in B. subtilis (Romero et al., 2011) we expressed sipW-tas $A$ or sipW-calY in a $B$. subtilis strain lacking the entire operon $\triangle$ tap $A_{o p}$; this strategy eliminated expression of TapA, a protein with no ortholog in $B$. cereus but retained the native sip $W$ gene. We observed that sip $W$-calY did not restore the wild-type phenotype (Figure 5, top row; Figures 6A,B top row), and sip W-tas $A$ complementation resulted in the formation of pellicles but not wrinkles (Figure 5, top row) and restored adhesion to abiotic surfaces (Figures 6A,B top row).

These results suggested that $B$. subtilis tapA might affect pellicle formation in the $B$. subtilis $\triangle$ tas $A$ mutant complemented with $B$. cereus sip $W$-tas $A$. Therefore, we examined pellicle formation in a $B$. subtilis $\triangle$ tapA mutant strain complemented with the following $B$. cereus regions: sip $W$-to-calY, sipW-tas $A$, or sipW-calY (Figure S2, top and middle rows). We observed that expression of sipW-to-calY was required to restore formation of wrinkly pellicles and adhesion to abiotic surfaces. Expression of sipW-tasA partially rescued the mutant phenotype, but sip $W$-calY failed to restore any of these phenotypes. Together, these observations confirmed the intrinsic ability of the protein products of the $B$. cereus sipW-to-calY region to facilitate the formation of wrinkly pellicles in the surrogate host B. subtilis. Additionally, TasA and CalY might have complementary roles in this phenotype, but the function of TasA is predominant.

\section{B. CEREUS TASA MIMICS THE FORMATION OF AMYLOID-LIKE FIBERS IN B. SUBTILIS BIOFILMS}

Two observations indicated that $B$. cereus TasA has amyloidlike properties: (i) B. cereus cells contained polymerized fibers (Figure 3), and (ii) the thin pellicles in B. cereus cells expressing TasA were stained with Congo Red (Figure 4). Based on these observations, we proposed that $B$. cereus TasA would display

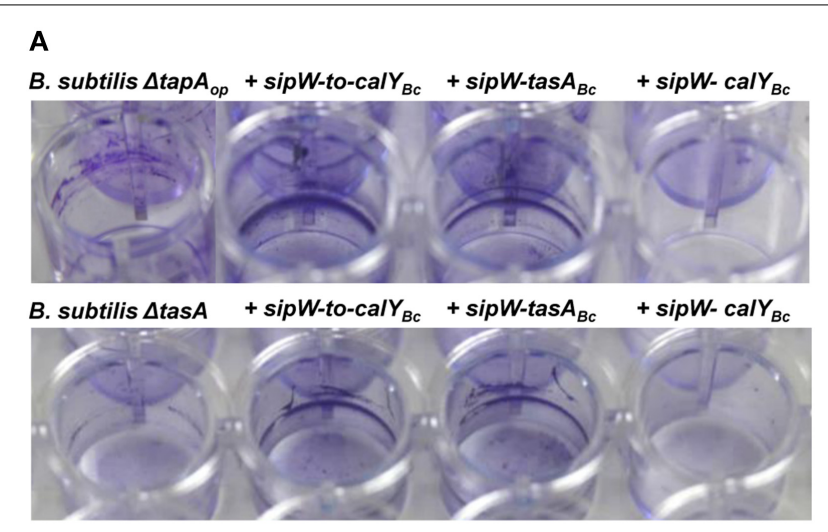

B

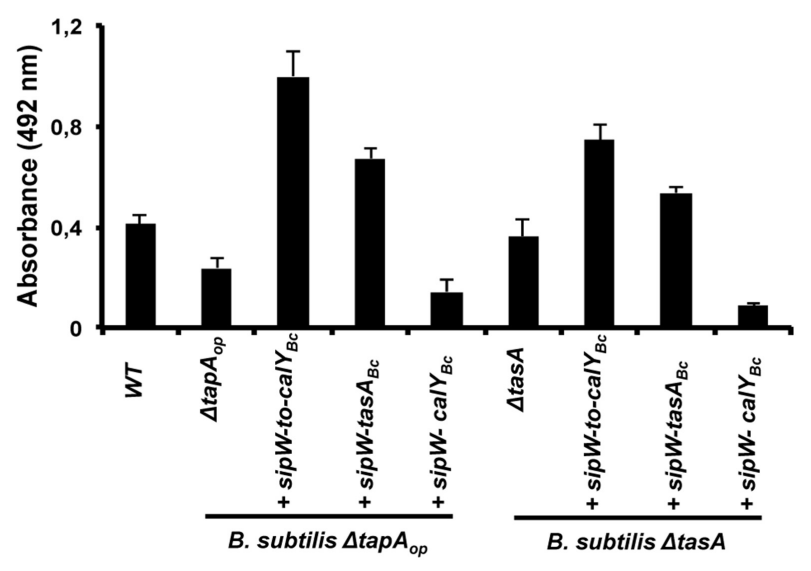

FIGURE 6 | Heterologous expression of B. cereus sipW-to-tas $A$ or $\operatorname{sip} W$-tas $A$ in $B$. subtilis enhances adhesion to abiotic surfaces. $B$. cereus alleles were ectopically integrated at the lacA or amyE locus of B. subtilis mutants lacking the entire tapA operon (B. subtilis $\triangle \operatorname{tap} A_{o p}$ ) or lacking tas $A$ alone ( $B$. subtilis $\Delta \operatorname{tas} A$ ); expression of these alleles was driven by an IPTG-inducible promoter. Biofilm experiments were performed in static cultures in MSgg broth and induced with 1 mM IPTG in 24-well plates. (A) Adhesion to abiotic surfaces was examined by staining the cultures with Crystal Violet, and pictures were obtained after $48 \mathrm{~h}$ of incubation at $30^{\circ} \mathrm{C}$. (B) Quantification of the amount of crystal violet retained in bacterial biomass. Data are the average and standard deviation from four independent experiments consisting of six internal replicates each.

similar behavior and aggregate into amyloid-like fibers when expressed in the surrogate host B. subtilis.

To test this hypothesis, we grew biofilms from mutant $B$. subtilis cells and cells complemented with different $B$. cereus loci; the cells were grown in TY medium supplemented with the amyloidspecific dye Congo Red (Figure 7). As expected, the B. subtilis $\triangle t a p A_{o p}$ and $\triangle$ tas $A$ mutants did not bind this dye. Staining with Congo Red was stronger in pellicles of B. subtilis $\Delta t a p A_{o p}$ mutant cells complemented with the entire sip $W$-to-calY region of B. cereus (Figure 7, top row); a strain that, as described above, formed wrinkly pellicles (Figure 5). The pellicles of $B$. subtilis $\triangle t a p A_{o p}$ strains expressing either sipW-tas $A$ or $\operatorname{sip} W$-calY were stained at low levels (Figure 7, top row). Notably, complementation of the B. subtilis $\triangle$ tas A mutant strain with the sipW-tas $A$ region or sipW-to-calY resulted in similar levels of Congo Red 


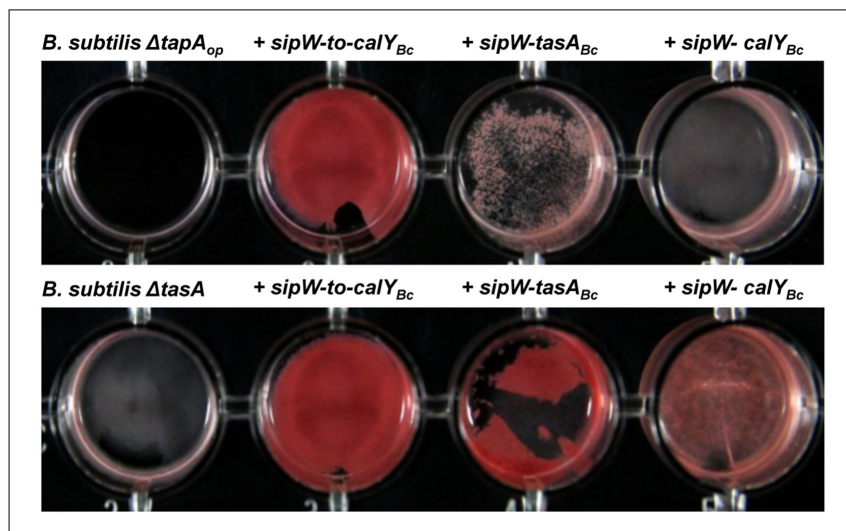

FIGURE 7 | Pellicles of $B$. subtilis complemented with sipW-to-tas $A$ or sipW-tas $A$ of $B$. cereus stained with specific amyloid dye Congo Red. $B$. cereus alleles were ectopically integrated at the lacA or amyE locus of B. subtilis mutants lacking the entire $\operatorname{tap} A$ operon (B. subtilis $\triangle \operatorname{tap} A_{o p}$ ) or lacking tas $A$ alone $(B$. subtilis $\Delta \operatorname{tas} A)$; expression of these alleles was driven by an IPTG-inducible promoter. Biofilm experiments were performed in static cultures in Ty-Congo Red $(20 \mu \mathrm{g} / \mathrm{ml})$-Coomassie Blue $(20 \mu \mathrm{g} / \mathrm{ml})$ broth and induced with $1 \mathrm{mM}$ IPTG in 24-well plates. Top-view pictures of pellicles after $24 \mathrm{~h}$ of growth at $30^{\circ} \mathrm{C}$ with no agitation.

staining; however, complementation with the sipW-calY construct resulted in weaker staining (Figure 7, bottom row). These data were consistent with the results obtained on pellicle formation and suggested that $B$. subtilis tapA influences cellular staining properties. However, when these experiments were performed using a $B$. subtilis $\triangle t a p A$ mutant strain, complementation with the entire $B$. cereus sipW-to-calY or sip $W$-tas $A$ but not sipW-calY restored Congo Red binding (Figure S2, bottom row).

To further examine the amyloid nature of $B$. cereus Tas A and CalY, we studied fibrillation of TasA and CalY on the B. subtilis cell surfaces (Figure 8). Transmission electron microscopy analysis revealed that $B$. subtilis $\Delta$ tas $A_{o p}$ and $\Delta$ tas $A$ mutant cells contained no fibers. Consistent with other experiments $B$. subtilis $\Delta$ tas $A_{o p}$ cells complemented with the $B$. cereus sipW-to-calY or sipWtas $A$ loci appeared decorated with several fibers (Figure 8, top row). However, complementation with sip W-calY, which did not restore Congo Red binding, also failed to promote fiber formation (Figure 8, top row). Expression of the entire B. cereus sipW-tocalY region or sip $W$-tas $A$ resulted in the formation of abundant fibers on the surfaces of $B$. subtilis $\triangle$ tas $A$ cells; however, expression of the sip $W$-calY promoted less abundant and thinner fibrils formation (Figure 8, bottom row). Finally in B. subtilis $\triangle$ tapA mutant; fibers were formed in $\triangle t a p A$ cells complemented with the entire region or with sipW-tas $A$ but not with sipW-calY (Figure S3). These results indicated that (i) the entire region of $B$. cereus drives the formation of fibers with amyloid properties similar to the $\operatorname{tap} A_{o p}$ of $B$. subtilis but does not require a tapA ortholog, (ii) B. cereus TasA and a at lesser extent CalY polymerizes to form fibers similar to B. subtilis TasA, and (iii) CalY complements the function of TasA in the formation of pellicles and fibers.

\section{DISCUSSION}

The bacterium Bacillus cereus is widely distributed in nature, and several species within this group inhabit soils, colonize arthropod guts or are pathogenic to humans (Bottone, 2010). The intrinsic factors contributing to this versatile ecological distribution include spore and biofilm formation (Auger et al., 2009; Elhariry, 2011). Spores are highly resistant to environmental stresses and are extremely adhesive, facilitating attachment to abiotic and biotic surfaces (Ball et al., 2008; Shaheen et al., 2010). Biofilms are considered to promote adhesion and protect cells from antimicrobials and other external insults and are thus difficult to eradicate (Flemming and Wingender, 2010). In this study, we examined the role of a specific genomic region in $B$. cereus biofilm formation.

Studies on biofilms of the phylogenetically related organism B. subtilis have elucidated both the genetic circuits that govern the biofilm developmental program and the structural components that facilitate assembly of the extracellular matrix (Romero, 2013; Vlamakis et al., 2013). The important matrix components of B. subtilis biofilms include exopolysaccharides, the hydrophobin BlsA and the amyloid-like protein TasA (Branda et al., 2004; Romero et al., 2010; Ostrowski et al., 2011). TasA, TapA and the signal peptidase SipW are especially important for correct assembly of the extracellular matrix. The studied B. cereus genomic region contains two TasA orthologs, TasA and CalY, and an ortholog of the signal peptidase SipW. However, this region lacks the accessory protein TapA. The fact that the expression of sip $W$, tas $A$ and calY of B. cereus are under control of the biofilm master regulator SinR (Fagerlund et al., 2014) led to think in their implication in biofilm formation, as the tap $A_{o p}$ in B. subtilis (Chu et al., 2006), and our data are supportive of this hypothesis (Figure 2). The divergent patterns of biofilm formation in tas $A$ and calY mutants suggested that these proteins function in different stages of biofilm formation: CalY might be more important for initial attachment, and TasA might be required for further maturation. Indeed, pioneer studies demonstrated that CalY could be purified from B. cereus cells at mi-log phase of growth (Fricke et al., 2001). In the other hand, this observation is not unprecedented; the interplay of diverse factors in biofilm formation can be observed in other bacteria species. The Gram-negative bacterium Pseudomonas putida contains two protein adhesins, LapF and LapA, which are essential for the initiation and maturation of biofilms, respectively (Martinez-Gil et al., 2010). We further propose that CalY and TasA are required for cell-to-cell and cellto-abiotic surface interactions. However, crystal violet staining and heterologous expression analyses in B. subtilis cells revealed that TasA might be more important for the interaction to abiotic surfaces (Figure 6).

The $B$. cereus tas $A$ mutant cells form an early biomass ring that is loosely bound to abiotic surfaces; therefore, other extracellular matrix components might be over-expressed. Two possible candidates are an exopolysaccharide or CalY. In B. subtilis, the absence of TasA increases the expression of the exopolysaccharide by an unknown regulatory pathway (Vlamakis et al., 2008), and a similar imbalance in the expression of components of the extracellular matrix has been reported in P. putida mutants lacking its large adhesin proteins (Martinez-Gil et al., 2013). Consistent with this observation, pellicles of a $B$. subtilis $\triangle$ tas $A$ mutant were easy to disrupt and fluid; expression of $B$. cereus sip $W$ tas $A$ suppressed these defects, and the pellicles resembled those of wild-type B. subtilis cells (Figure 5), indicating that EPS levels might be restored. The genome of $B$. cereus contains a region 

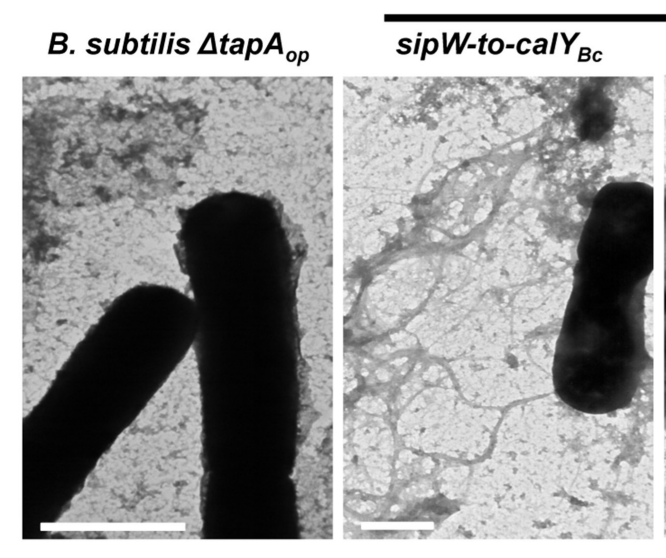

\section{B. subtilis $\triangle$ tap $A_{\text {op }}$}

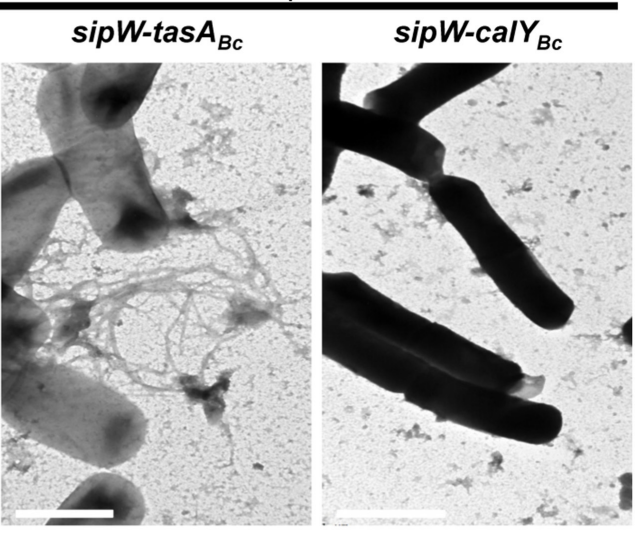

\section{B. subtilis $\triangle$ tas $A$}
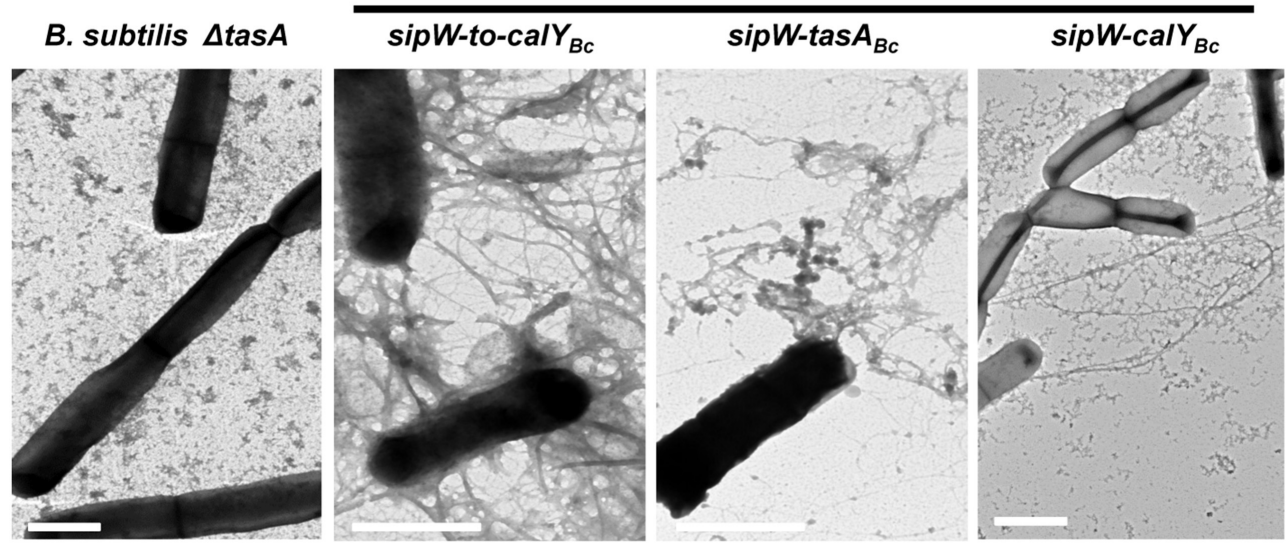

FIGURE 8 | Expression of $B$. cereus sipW-tasA leads to fiber formation in B. subtilis. $B$. cereus alleles were ectopically integrated the lacA or amyE locus of $B$. subtilis mutants lacking the entire $\operatorname{tap} A$ operon (B. subtilis

driven by an IPTG-inducible promoter. The strains were grown in MSgg broth and induced with $1 \mathrm{mM} \mathrm{IPTG}$ without shaking at $30^{\circ} \mathrm{C}$. Samples were collected after $24 \mathrm{~h}$, contrasted using uranyl acetate and then analyzed using transmission electron microscopy. Scale bars $=1 \mu \mathrm{m}$.

(BC_5267 to BC_5278) that highly resembles the operon dedicated to the synthesis of EPS in B. subtilis, but contrary to this bacteria species, the loci of $B$. cereus do not appear to be part of the $\sin R$ regulon (Fagerlund et al., 2014). Whether these or additional unknown factors of $B$. cereus are involved in biofilm formation needs to be clarified.

To build the extracellular matrix, B. subtilis TasA forms resistant fibers with amyloid properties. This process requires an accessory protein TapA, which contributes to the initiation and growth of TasA fibers (Romero et al., 2011, 2014). Our data from mutagenesis in $B$. cereus and heterologous expression in $B$. subtilis indicate that the $B$. cereus sip $W$-to-calY region contains all elements required for fiber assembly (Figures 3, 8). We propose that as previously described in B. subtilis, SipW functions as a signal peptidase that processes TasA and CalY to their mature forms for secretion (Tjalsma et al., 1998; Stover and Driks, 1999a,b). The rationale for this hypothesis is as follows: first, both proteins contain signal peptides with a canonical sequence that is a substrate for SipW proteolytic activity (Tjalsma et al., 1998; Terra et al., 2012); second, a sipW mutant is completely defective for biofilm formation (Figure 2); third, B. cereus TasA and CalY are not functional in B. subtilis unless they are coexpressed with the cognate SipW protein (Figure 5 and Figure $\mathrm{S} 1$ ). Our data suggest that B. cereus TasA is the more important for fiber formation than the other B. subtilis-TasA ortholog CalY. Abundant TasA fibers are present in wild-type and calY mutant B. cereus cells (Figure 3). Furthermore, in heterologous expression experiments, $B$. subtilis mutants lacking tapA formed fibers using $B$. cereus TasA (Figure 8). CalY has $62 \%$ identity with B. cereus TasA; CalY assembled into thin fibrils in B. cereus but failed to form fibers in B. subtilis unless TapA was present. One interpretation is that TapA of B. subtilis is able to cross seed the assembly of CalY fibers, a phenomenon recently reported in the assembly of the amyloid-like fiber Curli among Eschirichia coli and Salmonella typhimurium (Zhou et al., 2012).

Besides all our observations, we do not exclude the possibility that CalY has amyloid-like properties. Previous studies have shown that CalY is unusually resistant to SDS and heat treatments, and display high aggregative properties in organic solvent (Fricke et al., 2001); features associated with but not exclusive to amyloid proteins (Greenwald and Riek, 2010). The high sequence identity of CalY and TasA leads to the following 
mutually exclusive models: (i) CalY and TasA cooperate to assemble robust and stable fibers with amyloid properties including binding Congo Red, as described among CsgA and CsgB in assembly of Curli in E. coli (Shu et al., 2012); (ii) TasA and CalY form fibers independently, but these two types of fibers are important for biofilm assembly during different environmental conditions. An example of the diversification of amyloid-like proteins is the Gram-positive bacterium Streptomyces coelicolor, which has up to eight different chaplin proteins with a propensity to assemble amyloid-like fibers; this suggests the existence of significant plasticity to ensure the completion of complex developmental programs (Di Berardo et al., 2008; Sawyer et al., 2011). Further biochemical and morphological analyses of purified TasA and CalY are required to elucidate their amyloid properties.

In summary, we identified a specific $B$. cereus genomic region, which contains two independent genetic factors, the two-gene operon sipW-tas $A$ and the gene calY, which are both necessary for $B$. cereus biofilm formation. Directed mutagenesis in $B$. cereus and heterologous expression of the B. cereus alleles in B. subtilis revealed that TasA and, to a lesser extent, CalY have the intrinsic ability to polymerize and form fibers that are microscopically similar to the TasA amyloid-like fibers of B. subtilis. Finally, the Congo Red-binding ability of pellicles in TasA-expressing B. cereus cells and in B. subtilis cells complemented with sipW-tas $A$ and CalY or only sip $W$-tas $A$ point toward the amyloid nature of fibers formed by TasA.

\section{ACKNOWLEDGMENTS}

We would like to thank Gregorio Martín-Caballero and Adolfo Martínez for help and guidance in transmission electron microscopy. We thank Dr. Mirian Domenech for help in the construction of some strains. We thank Dr. Adam Driks for kindly providing antibodies against TasA. This work was funded by grant from Plan Nacional de I+D+I of Ministerio de Economía y Competitividad (AGL-2012-31968) co-financed by FEDER funds (European Union). Joaquín Caro is the recipient of a FPI contract from Ministerio de Economía y Competitividad and Diego Romero is funded by the program Ramón y Cajal (RyC-2011080605) from Ministerio de Economía y Competitividad and also co-financed by FEDER funds.

\section{SUPPLEMENTARY MATERIAL}

The Supplementary Material for this article can be found online at: http://www.frontiersin.org/journal/10.3389/fmicb. 2014.00745/abstract

\section{REFERENCES}

Arnaud, M., Chastanet, A., and Debarbouille, M. (2004). New vector for efficient allelic replacement in naturally nontransformable, low-GC-content, gram-positive bacteria. Appl. Environ. Microbiol. 70, 6887-6891. doi: 10.1128/AEM.70.11.6887-6891.2004

Auger, S., Ramarao, N., Faille, C., Fouet, A., Aymerich, S., and Gohar, M. (2009). Biofilm formation and cell surface properties among pathogenic and nonpathogenic strains of the Bacillus cereus group. Appl. Environ. Microbiol. 75, 6616-6618. doi: 10.1128/AEM.00155-09

Ball, D. A., Taylor, R., Todd, S. J., Redmond, C., Couture-Tosi, E., Sylvestre, P., et al. (2008). Structure of the exosporium and sublayers of spores of the Bacillus cereus family revealed by electron crystallography. Mol. Microbiol. 68, 947-958. doi: $10.1111 / j .1365-2958.2008 .06206 . x$
Berger, C. N., Sodha, S. V., Shaw, R. K., Griffin, P. M., Pink, D., Hand, P., et al. (2010). Fresh fruit and vegetables as vehicles for the transmission of human pathogens. Environ. Microbiol. 12, 2385-2397. doi: 10.1111/j.14622920.2010.02297.x

Blanco, L. P., Evans, M. L., Smith, D. R., Badtke, M. P., and Chapman, M. R. (2012). Diversity, biogenesis and function of microbial amyloids. Trends Microbiol. 20, 66-73. doi: 10.1016/j.tim.2011.11.005

Bottone, E. J. (2010). Bacillus cereus, a volatile human pathogen. Clin. Microbiol. Rev. 23, 382-398. doi: 10.1128/CMR.00073-09

Branda, S. S., Chu, F., Kearns, D. B., Losick, R., and Kolter, R. (2006). A major protein component of the Bacillus subtilis biofilm matrix. Mol. Microbiol. 59, 1229-1238. doi: 10.1111/j.1365-2958.2005.05020.x

Branda, S. S., Gonzalez-Pastor, J. E., Ben-Yehuda, S., Losick, R., and Kolter, R. (2001). Fruiting body formation by Bacillus subtilis. Proc. Natl. Acad. Sci. U.S.A. 98, 11621-11626. doi: 10.1073/pnas. 191384198

Branda, S. S., Gonzalez-Pastor, J. E., Dervyn, E., Ehrlich, S. D., Losick, R., and Kolter, R. (2004). Genes involved in formation of structured multicellular communities by Bacillus subtilis. J. Bacteriol. 186, 3970-3979. doi: 10.1128/JB.186.12.3970-3979.2004

Branda, S. S., Vik, S., Friedman, L., and Kolter, R. (2005). Biofilms: the matrix revisited. Trends Microbiol. 13, 20-26. doi: 10.1016/j.tim.2004.11.006

Burnett, S. L., and Beuchat, L. R. (2001). Human pathogens associated with raw produce and unpasteurized juices, and difficulties in decontamination. J. Ind. Microbiol. Biotechnol. 27, 104-110. doi: 10.1038/sj/jim/7000199

Carlin, F., Girardin, H., Peck, M. W., Stringer, S. C., Barker, G. C., Martinez, A., et al. (2000). Research on factors allowing a risk assessment of spore-forming pathogenic bacteria in cooked chilled foods containing vegetables: a FAIR collaborative project. Int. J. Food Microbiol. 60, 117-135. doi: 10.1016/S01681605(00)00304-4

Chapman, M. R., Robinson, L. S., Pinkner, J. S., Roth, R., Heuser, J., Hammar, M., et al. (2002). Role of Escherichia coli curli operons in directing amyloid fiber formation. Science 295, 851-855. doi: 10.1126/science.1067484

Chu, F., Kearns, D. B., Branda, S. S., Kolter, R., and Losick, R. (2006). Targets of the master regulator of biofilm formation in Bacillus subtilis. Mol. Microbiol. 59, 1216-1228. doi: 10.1111/j.1365-2958.2005.05019.x

De Jong, W., Wosten, H. A., Dijkhuizen, L., and Claessen, D. (2009). Attachment of Streptomyces coelicolor is mediated by amyloidal fimbriae that are anchored to the cell surface via cellulose. Mol. Microbiol. 73, 1128-1140. doi: 10.1111/j.13652958.2009.06838.x

Di Berardo, C., Capstick, D. S., Bibb, M. J., Findlay, K. C., Buttner, M. J., and Elliot, M. A. (2008). Function and redundancy of the chaplin cell surface proteins in aerial hypha formation, rodlet assembly, and viability in Streptomyces coelicolor. J. Bacteriol. 190, 5879-5889. doi: 10.1128/JB.00685-08

Dueholm, M. S., Petersen, S. V., Sonderkaer, M., Larsen, P., Christiansen, G., Hein, K. L., et al. (2010). Functional amyloid in Pseudomonas. Mol. Microbiol. 77, 1009-1020. doi: 10.1111/j.1365-2958.2010.07269.x

Elhariry, H. M. (2011). Attachment strength and biofilm forming ability of Bacillus cereus on green-leafy vegetables: cabbage and lettuce. Food Microbiol. 28, 1266-1274. doi: 10.1016/j.fm.2011.05.004

Epstein, E. A., and Chapman, M. R. (2008). Polymerizing the fibre between bacteria and host cells: the biogenesis of functional amyloid fibres. Cell. Microbiol. 10, 1413-1420. doi: 10.1111/j.1462-5822.2008.01148.x

Fagerlund, A., Dubois, T., Okstad, O. A., Verplaetse, E., Gilois, N., Bennaceur, I., et al. (2014). SinR controls enterotoxin expression in Bacillus thuringiensis biofilms. PLoS ONE 9:e87532. doi: 10.1371/journal.pone.00 87532

Flemming, H. C., and Wingender, J. (2010). The biofilm matrix. Nat. Rev. Microbiol. 8, 623-633. doi: 10.1038/nrmicro2415

Fowler, D. M., Koulov, A. V., Balch, W. E., and Kelly, J. W. (2007). Functional amyloid-from bacteria to humans. Trends Biochem. Sci. 32, 217-224. doi: 10.1016/j.tibs.2007.03.003

Fricke, B., Drossler, K., Willhardt, I., Schierhorn, A., Menge, S., and Rucknagel, P. (2001). The cell envelope-bound metalloprotease (camelysin) from Bacillus cereus is a possible pathogenic factor. Biochim. Biophys. Acta 1537, 132-146. doi: 10.1016/S0925-4439(01)00066-7

Greenwald, J., and Riek, R. (2010). Biology of amyloid: structure, function, and regulation. Structure 18, 1244-1260. doi: 10.1016/j.str.2010.08.009

Hobley, L., Ostrowski, A., Rao, F. V., Bromley, K. M., Porter, M., Prescott, A. R., et al. (2013). BslA is a self-assembling bacterial hydrophobin that coats the 
Bacillus subtilis biofilm. Proc. Natl. Acad. Sci. U.S.A. 110, 13600-13605. doi: 10.1073/pnas. 1306390110

Houry, A., Briandet, R., Aymerich, S., and Gohar, M. (2010). Involvement of motility and flagella in Bacillus cereus biofilm formation. Microbiology 156, 1009-1018. doi: 10.1099/mic.0.034827-0

Hsueh, Y. H., Somers, E. B., Lereclus, D., and Wong, A. C. (2006). Biofilm formation by Bacillus cereus is influenced by $\mathrm{PlcR}$, a pleiotropic regulator. Appl. Environ. Microbiol. 72, 5089-5092. doi: 10.1128/AEM.00573-06

Kamga Wambo, G. O., Burckhardt, F., Frank, C., Hiller, P., Wichmann-Schauer, H., Zuschneid, I., et al. (2011). The proof of the pudding is in the eating: an outbreak of emetic syndrome after a kindergarten excursion, Berlin, Germany, December 2007. Euro Surveill. 16:19839.

Karunakaran, E., and Biggs, C. A. (2011). Mechanisms of Bacillus cereus biofilm formation: an investigation of the physicochemical characteristics of cell surfaces and extracellular proteins. Appl. Microbiol. Biotechnol. 89, 1161-1175. doi: 10.1007/s00253-010-2919-2

Kobayashi, K., and Iwano, M. (2012). BslA(YuaB) forms a hydrophobic layer on the surface of Bacillus subtilis biofilms. Mol. Microbiol. 85, 51-66. doi: 10.1111/j.1365-2958.2012.08094.x

Lindback, T., Mols, M., Basset, C., Granum, P. E., Kuipers, O. P., and Kovacs, A. T. (2012). CodY, a pleiotropic regulator, influences multicellular behaviour and efficient production of virulence factors in Bacillus cereus. Environ. Microbiol. 14, 2233-2246. doi: 10.1111/j.1462-2920.2012.02766.x

López, D., Vlamakis, H., Losick, R., and Kolter, R. (2009). Paracrine signaling in a bacterium. Genes Dev. 23, 1631-1638. doi: 10.1101/gad.1813709

Martinez-Gil, M., Quesada, J. M., Ramos-Gonzalez, M. I., Soriano, M. I., De Cristobal, R. E., and Espinosa-Urgel, M. (2013). Interplay between extracellular matrix components of Pseudomonas putida biofilms. Res. Microbiol. 164, 382-389. doi: 10.1016/j.resmic.2013.03.021

Martinez-Gil, M., Yousef-Coronado, F., and Espinosa-Urgel, M. (2010). LapF, the second largest Pseudomonas putida protein, contributes to plant root colonization and determines biofilm architecture. Mol. Microbiol. 77, 549-561. doi: 10.1111/j.1365-2958.2010.07249.x

McKillip, J. L. (2000). Prevalence and expression of enterotoxins in Bacillus cereus and other Bacillus spp., a literature review. Antonie Van Leeuwenhoek 77, 393-399. doi: 10.1023/A:1002706906154

Ostrowski, A., Mehert, A., Prescott, A., Kiley, T. B., and Stanley-Wall, N. R. (2011). YuaB functions synergistically with the exopolysaccharide and TasA amyloid fibers to allow biofilm formation by Bacillus subtilis. J. Bacteriol. 193, 4821-4831. doi: 10.1128/JB.00223-11

O’toole, G. A., Pratt, L. A., Watnick, P. I., Newman, D. K., Weaver, V. B., and Kolter, R. (1999). Genetic approaches to study of biofilms. Meth. Enzymol. 310, 91-109. doi: 10.1016/S0076-6879(99)10008-9

Pasvolsky, R., Zakin, V., Ostrova, I., and Shemesh, M. (2014). Butyric acid released during milk lipolysis triggers biofilm formation of Bacillus species. Int. J. Food Microbiol. 181, 19-27. doi: 10.1016/j.ijfoodmicro.2014.04.013

Pflughoeft, K. J., Sumby, P., and Koehler, T. M. (2011). Bacillus anthracis sin locus and regulation of secreted proteases. J. Bacteriol. 193, 631-639. doi: 10.1128/JB.01083-10

Romero, D. (2013). Bacterial determinants of the social behavior of Bacillus subtilis. Res. Microbiol. 164, 788-798. doi: 10.1016/j.resmic.2013.06.004

Romero, D., Aguilar, C., Losick, R., and Kolter, R. (2010). Amyloid fibers provide structural integrity to Bacillus subtilis biofilms. Proc. Natl. Acad. Sci. U.S.A. 107, 2230-2234. doi: 10.1073/pnas.0910560107

Romero, D., Vlamakis, H., Losick, R., and Kolter, R. (2011). An accessory protein required for anchoring and assembly of amyloid fibres in B. subtilis biofilms. Mol. Microbiol. 80, 1155-1168. doi: 10.1111/j.1365-2958.2011.07653.x

Romero, D., Vlamakis, H., Losick, R., and Kolter, R. (2014). Functional analysis of the accessory protein TapA in Bacillus subtilis amyloid fiber assembly. J. Bacteriol. 196, 1505-1513. doi: 10.1128/JB.01363-13

Sawyer, E. B., Claessen, D., Haas, M., Hurgobin, B., and Gras, S. L. (2011). The assembly of individual chaplin peptides from Streptomyces coelicolor into functional amyloid fibrils. PLOS ONE 6:e18839. doi: 10.1371/journal.pone.0018839
Schwartz, K., Syed, A. K., Stephenson, R. E., Rickard, A. H., and Boles, B. R. (2012). Functional amyloids composed of phenol soluble modulins stabilize Staphylococcus aureus biofilms. PLoS Pathog. 8:e1002744. doi: 10.1371/journal.ppat. 1002744

Shaheen, R., Svensson, B., Andersson, M. A., Christiansson, A., and SalkinojaSalonen, M. (2010). Persistence strategies of Bacillus cereus spores isolated from dairy silo tanks. Food Microbiol. 27, 347-355. doi: 10.1016/j.fm.2009.11.004

Shemesh, M., and Chai, Y. (2013). A combination of glycerol and manganese promotes biofilm formation in Bacillus subtilis via histidine kinase KinD signaling. J. Bacteriol. 195, 2747-2754. doi: 10.1128/JB.00028-13

Shu, Q., Crick, S. L., Pinkner, J. S., Ford, B., Hultgren, S. J., and Frieden, C. (2012). The E. coli CsgB nucleator of curli assembles to beta-sheet oligomers that alter the CsgA fibrillization mechanism. Proc. Natl. Acad. Sci. U.S.A. 109, 6502-6507. doi: $10.1073 /$ pnas. 1204161109

Stover, A. G., and Driks, A. (1999a). Control of synthesis and secretion of the Bacillus subtilis protein YqxM. J. Bacteriol. 181, 7065-7069.

Stover, A. G., and Driks, A. (1999b). Secretion, localization, and antibacterial activity of TasA, a Bacillus subtilis spore-associated protein. J. Bacteriol. 181, 1664-1672.

Terra, R., Stanley-Wall, N. R., Cao, G., and Lazazzera, B. A. (2012). Identification of Bacillus subtilis SipW as a bifunctional signal peptidase that controls surfaceadhered biofilm formation. J. Bacteriol. 194, 2781-2790. doi: 10.1128/JB. 06780-11

Thorsen, L., Azokpota, P., Munk Hansen, B., Ronsbo, M. H., Nielsen, K. F., Hounhouigan, D. J., et al. (2011). Formation of cereulide and enterotoxins by Bacillus cereus in fermented African locust beans. Food Microbiol. 28, 1441-1447. doi: 10.1016/j.fm.2011.07.003

Tjalsma, H., Bolhuis, A., Van Roosmalen, M. L., Wiegert, T., Schumann, W., Broekhuizen, C. P., et al. (1998). Functional analysis of the secretory precursor processing machinery of Bacillus subtilis: identification of a eubacterial homolog of archaeal and eukaryotic signal peptidases. Genes Dev. 12, 2318-2331. doi: 10.1101/gad.12.15.2318

Vilain, S., Pretorius, J. M., Theron, J., and Brozel, V. S. (2009). DNA as an adhesin: Bacillus cereus requires extracellular DNA to form biofilms. Appl. Environ. Microbiol. 75, 2861-2868. doi: 10.1128/AEM.01317-08

Vlamakis, H., Aguilar, C., Losick, R., and Kolter, R. (2008). Control of cell fate by the formation of an architecturally complex bacterial community. Genes Dev. 22, 945-953. doi: 10.1101/gad.1645008

Vlamakis, H., Chai, Y., Beauregard, P., Losick, R., and Kolter, R. (2013). Sticking together: building a biofilm the Bacillus subtilis way. Nat. Rev. Microbiol. 11, 157-168. doi: 10.1038/nrmicro2960

Zhou, Y., Smith, D., Leong, B. J., Brannstrom, K., Almqvist, F., and Chapman, M. R. (2012). Promiscuous cross-seeding between bacterial amyloids promotes interspecies biofilms. J. Biol. Chem. 287, 35092-35103. doi: 10.1074/jbc.M112.383737

Conflict of Interest Statement: The authors declare that the research was conducted in the absence of any commercial or financial relationships that could be construed as a potential conflict of interest.

Received: 01 November 2014; accepted: 09 December 2014; published online: 13 January 2015.

Citation: Caro-Astorga J, Pérez-García A, de Vicente A and Romero D (2015) A genomic region involved in the formation of adhesin fibers in Bacillus cereus biofilms. Front. Microbiol. 5:745. doi: 10.3389/fmicb.2014.00745

This article was submitted to Food Microbiology, a section of the journal Frontiers in Microbiology.

Copyright (c) 2015 Caro-Astorga, Pérez-García, de Vicente and Romero. This is an open-access article distributed under the terms of the Creative Commons Attribution License (CC BY). The use, distribution or reproduction in other forums is permitted, provided the original author(s) or licensor are credited and that the original publication in this journal is cited, in accordance with accepted academic practice. No use, distribution or reproduction is permitted which does not comply with these terms. 